\title{
Cloning of the Rice Xo1 Resistance Gene and Interaction of the Xo1 Protein with the Defense-Suppressing Xanthomonas Effector Tal2h
}

\author{
Andrew C. Read, ${ }^{1}$ Mathilde Hutin, ${ }^{1,2}$ Matthew J. Moscou, ${ }^{3}$ Fabio C. Rinaldi, ${ }^{1}$ and Adam J. Bogdanove ${ }^{1, \dagger}$ \\ ${ }^{1}$ Plant Pathology and Plant-Microbe Biology Section, School of Integrative Plant Science, Cornell University, Ithaca, NY 14853, \\ U.S.A. \\ ${ }^{2}$ IRD, CIRAD, Université Montpellier, IPME, 34000 Montpellier, France \\ ${ }^{3}$ The Sainsbury Laboratory, University of East Anglia, Norwich Research Park, Norwich, NR4 7UK, U.K. \\ Accepted 24 July 2020.
}

\begin{abstract}
The Xo1 locus in the heirloom rice variety Carolina Gold Select confers resistance to bacterial leaf streak and bacterial blight, caused by Xanthomonas oryzae pv. oryzicola and X. oryzae pv. oryzae, respectively. Resistance is triggered by pathogendelivered transcription activator-like effectors (TALEs) independent of their ability to activate transcription and is suppressed by truncated variants called truncTALEs, common among Asian strains. By transformation of the susceptible variety Nipponbare, we show that one of 14 nucleotide-binding, leucine-rich repeat (NLR) protein genes at the locus, with a zinc finger BED domain, is the $X o 1$ gene. Analyses of published transcriptomes revealed that the $X o 1$-mediated response is more similar to those mediated by two other NLR resistance genes than it is to the response associated with TALE-specific transcriptional activation of the executor resistance gene $\mathrm{Xa23}$ and that a truncTALE dampens or abolishes activation of defense-associated genes by Xo1. In Nicotiana benthamiana leaves, fluorescently tagged Xo1 protein, like TALEs and truncTALEs, localized to the nucleus. And endogenous Xo1 specifically coimmunoprecipitated from rice leaves with a
\end{abstract}

Andrew C. Read and Mathilde Hutin contributed equally.

Current address for F. C. Rinaldi: Vertex Pharmaceuticals, 50 Northern Avenue, Boston, MA 02210, U.S.A.

${ }^{\dagger}$ Corresponding author: A. J. Bogdanove; ajb7@cornell.edu

Funding: This work was supported by the Plant Genome Research Program of the National Science Foundation (Division of Integrative Organismal Systems IOS-1444511 to A. J. Bogdanove), the National Institute of Food and Agriculture of the United States Department of Agriculture (2018-67011-28025 to A. C. Read), and the Gatsby Charitable Foundation (to M. J. Moscou). We also acknowledge support from the National Institutes of Health to the Proteomics Facility for the Orbitrap Fusion mass spectrometer (shared instrumentation grant 1S10 OD017992-01) and to the Imaging Facility for the Zeiss LSM 710 confocal microscope (shared instrumentation grant S10RR025502).

*The $\boldsymbol{e}$-Xtra logo stands for "electronic extra" and indicates there are supplementary materials, supplementary tables, and a supplementary dataset published online.

The author(s) declare no conflict of interest.

() 2020 The American Phytopathological Society pathogen-delivered, epitope-tagged truncTALE. These observations suggest that suppression of Xo1-function by truncTALEs occurs through direct or indirect physical interaction. They further suggest that effector coimmunoprecipitation may be effective for identifying or characterizing other resistance genes.

Keywords: defense suppression, effectors, mass spectrometry, nucleotide binding leucine-rich repeat (NLR), protein-protein interaction, resistance genes, transcription activator-like effector (TALE), truncTALE

Bacterial leaf streak of rice caused by Xanthomonas oryzae pv. oryzicola is an increasing threat to production in many parts of the world, especially in Africa. Bacterial blight of rice caused by $X$. oryzae pv. oryzae has long been a major constraint in Asia and is becoming prevalent in Africa. The purified American heirloom rice variety Carolina Gold Select (hereafter Carolina Gold [McClung and Fjellstrom 2010]) is resistant to all tested African strains of $X$. oryzae pv. oryzicola and some tested strains of $X$. oryzae pv. oryzae (Read et al. 2016). Using an African strain of X. oryzae pv. oryzicola, resistance was mapped to chromosome 4 and designated as Xol (Triplett et al. 2016). Both X. oryzae pv. oryzicola and $X$. oryzae pv. oryzicola deploy multiple type III-secreted transcription activator-like effectors (TALEs) during infection. TALEs enter the plant nucleus and bind to promoters, each with different sequence specificity, to transcriptionally activate effector-specific target genes (Perez-Quintero and Szurek 2019). Some of these genes, called susceptibility genes, contribute to disease development (Hutin et al. 2015). In some host genotypes, a TALE may activate a so-called executor resistance gene, leading to host cell death that stops the infection (Bogdanove et al. 2010). Most of the cloned resistance genes for bacterial blight are, in fact, executor genes (Zhang et al. 2015). Xol is different. It mediates resistance in response to TALEs with distinct DNA-binding specificities independent of their ability to activate transcription (Triplett et al. 2016). Also, unlike executor genes, Xol function is suppressed by a truncated variant class of these effectors known as truncTALEs (also called iTALEs). Like TALEs, TruncTALEs nuclear localize (Ji et al. 2016), however, due to large $\mathrm{N}$ - and C-terminal deletions, they do not bind DNA (Read et al. 2016). 
Xol maps to a region that, in the reference rice genome (cv. Nipponbare), contains seven nucleotide-binding, leucine-rich repeat (NLR) protein genes (Triplett et al. 2016). NLR genes are the largest class of plant disease resistance genes. NLR proteins recognize specific, corresponding pathogen effector proteins through direct interaction or by detecting effectordependent changes of host target proteins and mediate downstream defense signaling that leads to expression of defense genes and a programmed localized cell death, the hypersensitive reaction (HR) (Lolle et al. 2020). Recently, by wholegenome sequencing, we determined that the Xol locus in Carolina Gold comprises 14 NLR genes. We identified one of these, $X_{o 1} 1_{11}$, as a strong candidate based on its structural similarity to the previously cloned and only known NLR resistance gene for bacterial blight, $\mathrm{Xal}$ (Read et al. 2020). Xal, originally identified in the rice variety Kogyoku, maps to the same location (Yoshimura et al. 1998) and behaves similarly to Xol: it mediates recognition of TALEs with distinct DNAbinding specificities (and thus confers resistance also to bacterial leaf streak), and its activity is suppressed by truncTALEs (Ji et al. 2016). $X_{o 1} 1_{11}$ and $X a 1$ are members of a small subfamily of NLR genes that encode an unusual N-terminal domain comprising a zinc finger BED (zfBED) motif (Read et al. 2020).

To ascertain whether $\mathrm{Xol}_{11}$ is the gene responsible for $\mathrm{Xol}$ resistance, we generated transgenic Nipponbare plants expressing it. For transformation, we amplified the genomic $\mathrm{Xol}_{11}$ coding sequence $(5,882 \mathrm{bp})$ as well as the 993 -bp region upstream of the start codon and cloned them together into a

\section{Xoc CFBP7331}

\section{Event 1

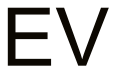 \\ $\mathrm{p} 2 \mathrm{~h}$
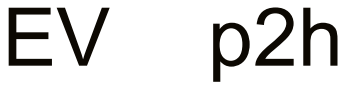
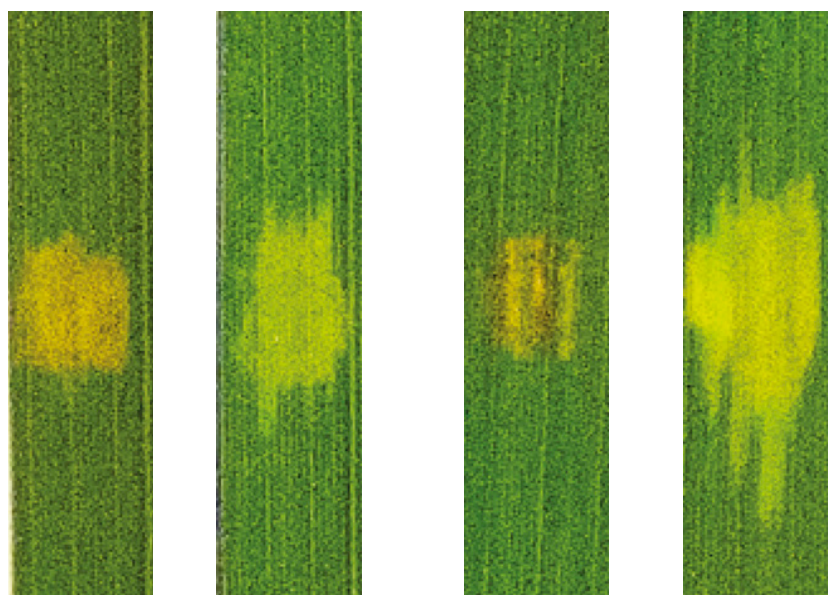

Fig. 1. Transgenic Nipponbare plants expressing $X o 1_{11}$ are resistant to African Xanthomonas oryzae pv. oryzicola CFBP7331 and the resistance is suppressed by a truncTALE (a variant of a transcription activator-like effector). Susceptible cultivar Nipponbare was transformed with pAR902 (Supplementary Materials), and leaves of T0 plants from two events were syringeinfiltrated with African strain $X$. oryzae pv. oryzicola CFBP7311 carrying either empty vector $(\mathrm{EV})$ or tal2h (p2h) adjusted to an optical density at 600 $\mathrm{nm}$ of 0.4 . Leaves were photographed on a light box at 4 days after inoculation. Resistance is apparent as a hypersensitive reaction (necrosis) at the site of inoculation and disease as expanded, translucent water-soaking. binary vector with a $35 \mathrm{~S}$ terminator. T0 $\mathrm{Xo1}_{11}$ plants were inoculated by syringe infiltration with African strain $X$. oryzae pv. oryzicola CFBP7331, which has no truncTALE of its own, carrying either an empty vector (EV) or the plasmid-borne truncTALE gene tal2h $(\mathrm{p} 2 \mathrm{~h})$ from the Asian strain $X$. oryzae pv. oryzicola BLS256 (Read et al. 2016). Phenotypes of CFBP7331(EV) and CFBP7331(p2h) were confirmed on untransformed Nipponbare and Carolina Gold plants (Supplementary Fig. S1). Plants from two $\mathrm{Xol}_{11}$ transformation events displayed resistance to the strain with the EV but not to the strain carrying tal2h (Fig. 1), demonstrating that $X o l_{11}$ is the Xol gene.

NLR protein activation is characteristically followed by a suite of responses that includes massive transcriptional reprogramming, leading both to $\mathrm{HR}$ and to activation of a large number of defense-associated genes (Cui et al. 2015). To gain insight into the nature of Xol-mediated resistance, we compared the global profile of differentially expressed genes (DEGs) during Xol-mediated defense to those of two other NLR genes in rice and to the profile associated with an executor gene. We used our previously reported RNA-seq data from Carolina Gold plants inoculated with CFBP7331(EV) or mock inoculum (Read et al. 2020), data for the NLR gene Pia for resistance to the rice blast pathogen Magnaporthe oryzae (Tanabe et al. 2014), data from rice resistant to bacterial leaf streak due to transgenic expression of the maize NLR gene Rxol (Xie et al. 2007; Zhou et al. 2010), and data for the transcriptomic response associated with induction of the executor resistance gene Xa23 by an X. oryzae pv. oryzicola strain with the corresponding TALE (Tariq et al. 2018). Though limited, these datasets include the only currently available expression data for NLR and executor gene-mediated resistance to Xanthomonas spp. in rice. DEGs ( $\log _{2}$-fold change $>1$ or $<-1 ; P$ value $>0.05)$ in the comparison between pathogeninoculated and mock-inoculated plants were compared across the four datasets. The total number of DEGs ranged from 10,050 for $\mathrm{Xol}$ to 628 for $\mathrm{Xa23}$, and the overall profiles were largely distinct (Fig. 2A; Supplementary Table S1). For each resistance gene, there were a number of DEGs found only in the pathogen-to-mock comparison for that dataset, and this was highest for Xol (7,121 genes) (Fig. 2A; Supplementary Table S1). Differences among the overall DEG profiles may be influenced by the expression assay (RNA-seq versus microarray), pathogen, annotation, or timepoints used. To compare the responses further the expression of 340 rice genes associated with plant defense response (gene ontology group 0006952) was examined. The Xol profile comprised the largest number of plant defense DEGs (99) and had more DEGs in common with the other NLR-mediated responses (16 with Rxol and 26 with $\mathrm{Pia}$ ) than with the executor gene response (8) (Fig. 2B). Additionally, each of the NLR-mediated responses resulted in a larger number of defense DEGs (26 for Rxo1, 41 for Pia) than the $X a 23$ response (14), and based on principal component analysis of the defense DEG profiles, were more similar to one another than to the executor gene response (Fig. 2B and C; Supplementary Table S2).

We also compared DEGs relative to mock in Carolina Gold plants inoculated with CFBP7331(EV) and Carolina Gold plants inoculated with CFBP7331(p2h) (Read et al. 2020), to gain insight into how Xol-mediated resistance is overcome by a pathogen delivering a truncTALE. In contrast to the 99 defense-response genes differentially expressed in response to CFBP7331(EV), only 18 defense genes were differentially expressed in response to CFBP7331(p2h) (Fig. 2D). Of these 18 genes, seven were differentially expressed only in the response to the strain with tal $2 h$, four up and three down. Of the remaining 11 , four were up and two were down in both responses, but each less so in the 
response to the strain with tal2h. The other five moved in opposite directions entirely, up in the absence but repressed in the presence of tal $2 h$, relative to mock. This expression profile during suppression of Xol-mediated resistance is consistent with Tal2h functioning early in the defense cascade. The bacterial leaf streak susceptibility gene OsSULTR3;6 (Cernadas et al. 2014), activated by Tal8e of CFBP7331 (Wilkins et al. 2015), is strongly induced by both CFBP7331(EV) and CFBP7331(p2h) (Supplementary Table S3), indicating that TALE function is not compromised by Xo1 or by Tal2h.

The observation that Xo1 reprograms transcription of canonical defense genes upon recognition of the cognate
A

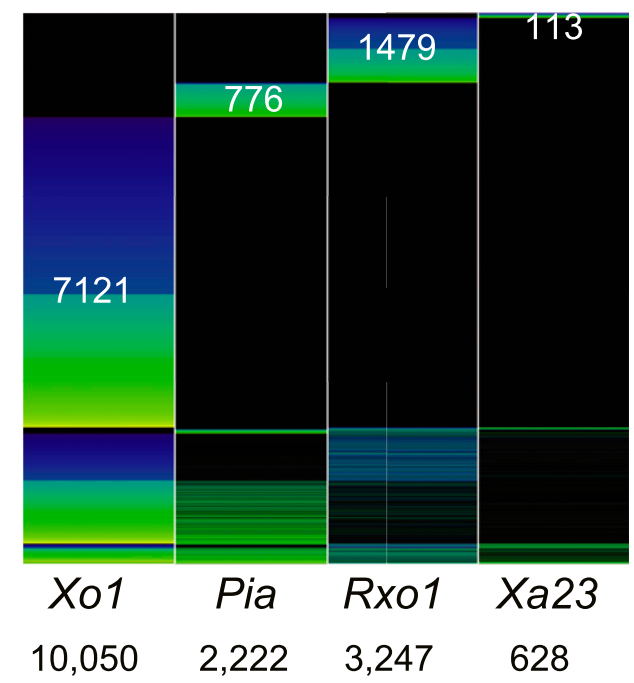

C Principal Component Analysis

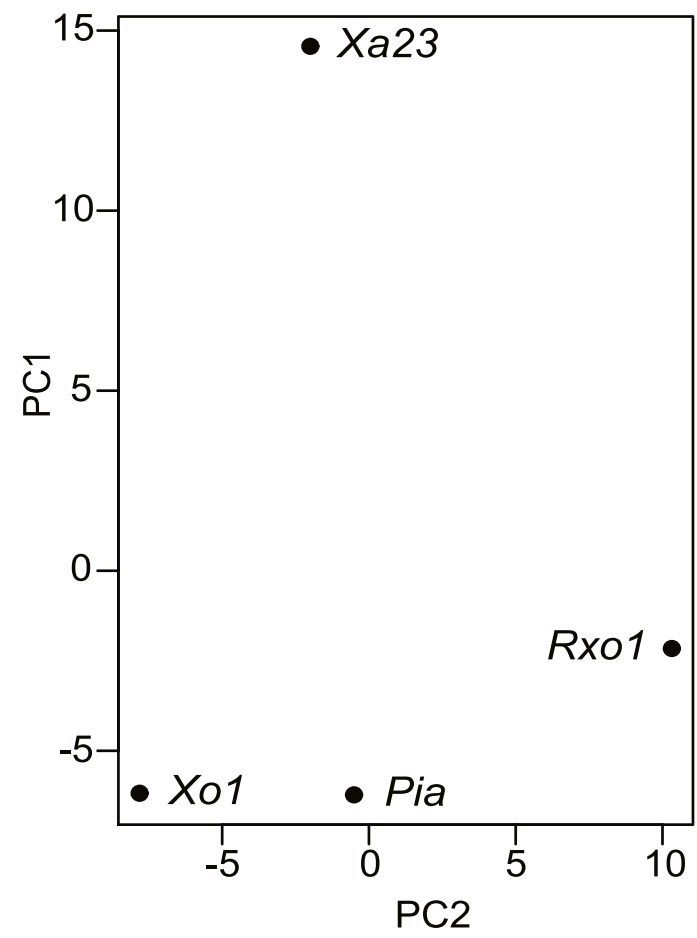

B

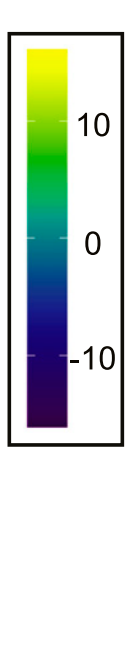

B GO:0006952 DEGs

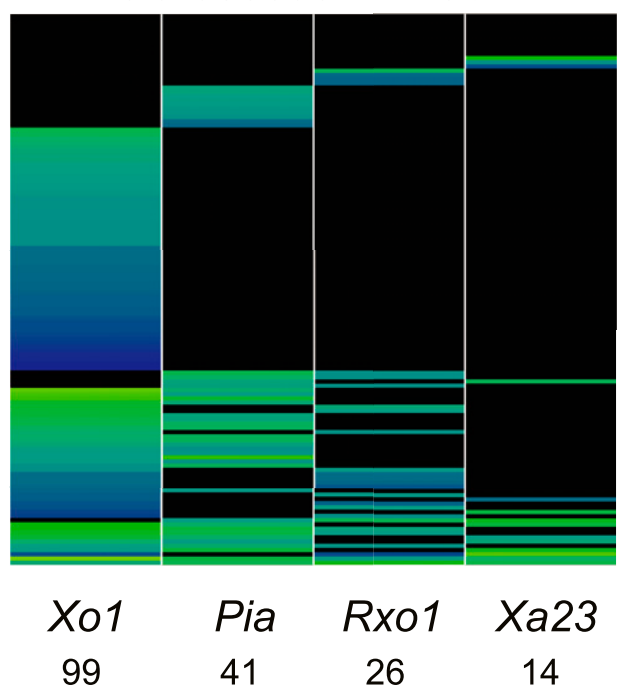

EV and $p 2 h$ DEGs

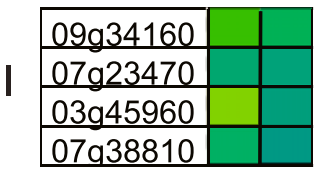

resistance protein

thaumatin

thaumatin

lectin receptor-type protein kinase

II

\begin{tabular}{|l|l|l|}
\hline $08 g 29370$ & & peptidyl-prolyl cis-trans isomerase \\
\hline $04 \mathrm{~g} 58710$ & & AMP-binding domain containing protein
\end{tabular}

$I I I$\begin{tabular}{|c|l|l|}
\hline $12 \mathrm{~g} 36880$ & \\
\hline $12 \mathrm{~g} 36850$ & & \\
\hline $03 \mathrm{~g} 55240$ & & \\
\hline $08 \mathrm{~g} 42800$ & & \\
\hline $01 \mathrm{~g} 06740$ & \\
\hline
\end{tabular}

pathogenesis-related Bet v I family pathogenesis-related Bet $v$ I family cytochrome P450 expressed protein ribosome inactivating protein

\begin{tabular}{|l|}
\hline $07 \mathrm{~g} 18750$ \\
\hline IV $01 \mathrm{~g} 68589$ \\
\hline $06 \mathrm{~g} 37690$ \\
\hline $02 \mathrm{~g} 41890$ \\
\hline
\end{tabular}

LTPL42 - Protease inhibitor/seed storage LTPL39 - Protease inhibitor/seed storage S-locus-like receptor protein kinase phytosulfokine receptor precursor

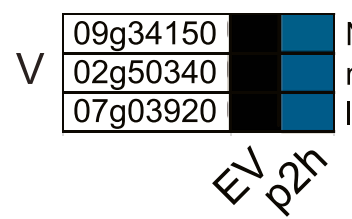

NBS-LRR disease resistance protein membrane attack/perforin/complement lectin-like receptor kinase 7

Fig. 2. The Xo1-mediated transcriptomic response is similar to those of other nucleotide-binding, leucine-rich repeat (NLR) genes and is essentially eliminated by Tal2h. A, Expression heatmaps (columns) showing all differentially expressed genes (DEGs) in plants undergoing the resistant response compared with mock-inoculated plants for Xol, the NLR genes Pia and Rxo1, and the executor resistance gene Xa23. White numbers for each on the heatmap indicate the number of DEGs specific to each response. Total numbers of DEGs are indicated below. B, Heatmaps for the subset of DEGs from A that belong to gene ontology group 0006952, defense response, with totals displayed at bottom. C, Principal component analysis. The first two principal components (PC1 and PC2) explain 54.0 and $31.6 \%$ of the variation with a total of $85.6 \%$. PC1 demarcated two major clusters: i) Xo1, Pia, and Rxo1 and ii) Xa23. D, Heatmaps for the 18 defense-response DEGs identified in the comparison of Carolina Gold plants inoculated with CFBP7331(p2h) to mock-inoculated plants. The "EV" heatmap shows their expression relative to mock in Carolina Gold plants inoculated with CFBP7331(EV) (resistance), and the "p2h" column shows their expression relative to mock in the presence of Tal2h (disease). The DEGs have been divided into five categories: I, induced in both; II, downregulated in both; III, induced in resistance and downregulated in disease; IV, not differentially expressed in resistance and induced in disease; and V, not differentially expressed in resistance and downregulated in disease. 
pathogen effector and that reprogramming by Xo1 is essentially blocked by Tal2h led us to explore whether Xo1 localizes to the same subcellular location as TALEs and truncTALEs. Some, but not all, NLR proteins nuclear localize (Caplan et al. 2008; Cheng et al. 2009; Shen et al. 2007; Wirthmueller et al. 2007), and we previously identified putative nuclear localization signals (NLSs) in $\mathrm{Xo1}_{11}$ (Read et al. 2020). We generated expression constructs for a green fluorescent protein (GFP) fusion to the $\mathrm{N}$ terminus of $\mathrm{Xo1}$ as well as an $\mathrm{N}$-terminal monomeric red fluorescent protein (mRFP) fusion both to a TALE (Tallc of $X$. oryzae pv. oryzicola BLS256) and to Tal2h. These constructs were delivered into Nicotiana benthamiana leaves using
Agrobacterium tumefaciens GV3101, and the leaves imaged with a Zeiss 710 confocal microscope (Fig. 3). GFP-Xo1 in the absence of either effector but with free mRFP localized to foci that appeared to be nuclei. Coexpression with mRFP-Tal1c or with mRFP-Tal2h confirmed that these foci were nuclei.

The localization of Xo1, the TALE, and the truncTALE to the nucleus when transiently expressed in $N$. benthamiana led us to pursue the hypothesis that Xo1 physically interacts with one or both of these proteins in the native context. We generated plasmid constructs that add a $3 \times$ FLAG tag to the C-terminus of TALE Tal1c or the truncTALE Tal2h (Tal1c-FLAG and Tal2h-FLAG) and introduced them individually into the
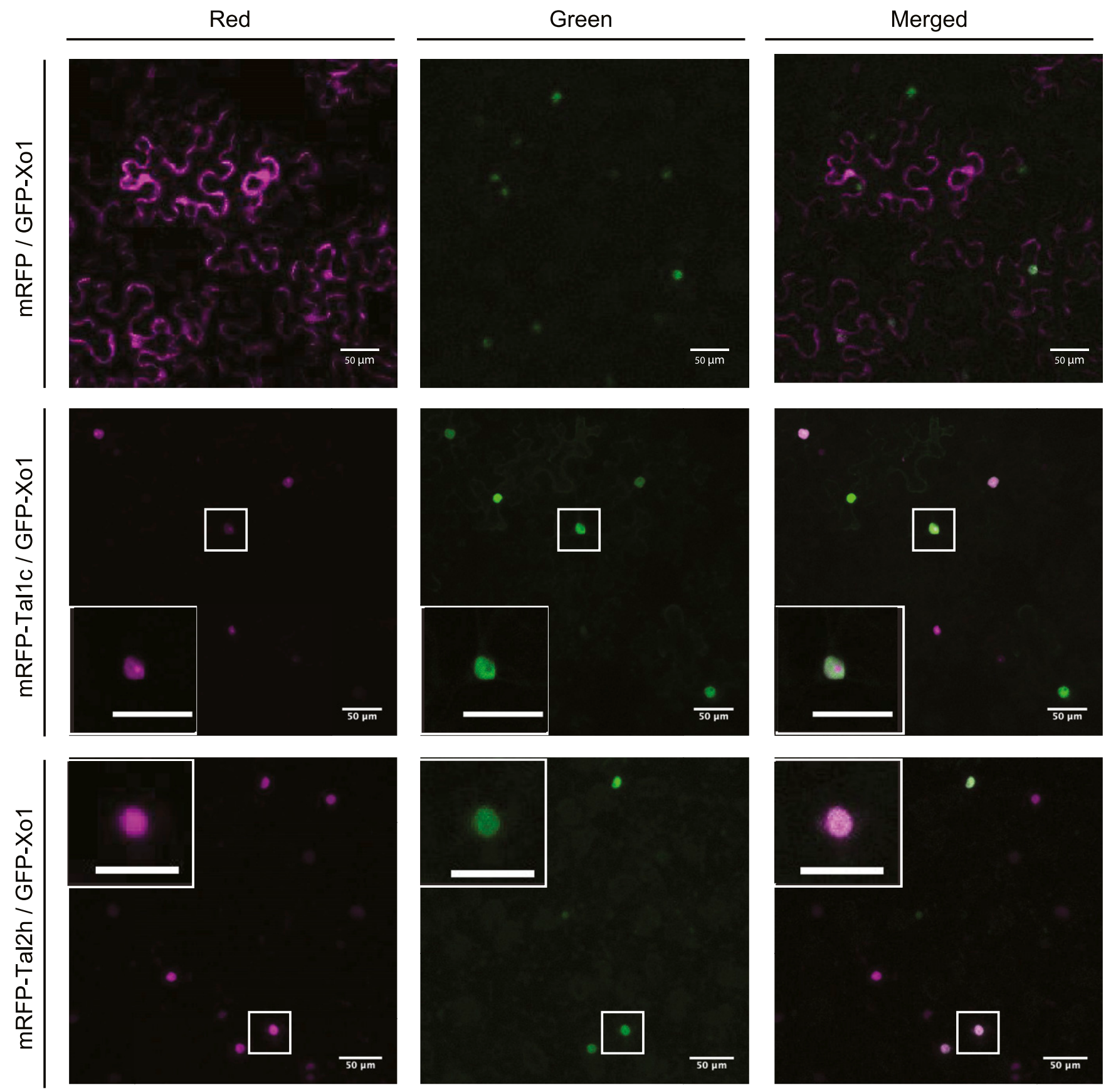

Fig. 3. Xo1 localizes to the nucleus. Using Agrobacterium coinfiltrations, an expression construct for Xo1 with green fluorescent protein (GFP) at the N terminus (GFP-Xo1) together with a p19 silencing-suppressor construct were introduced into Nicotiana benthamiana leaves alone, or with a construct for monomeric red fluorescent protein (mRFP), mRFP fused to transcription activator-like effector (TALE) Tal1c (mRFP-Tal1c), or mRFP fused to the truncTALE Tal2h (mRFP-Tal2h). Confocal image stacks were taken at 3 days after inoculation and are presented as maximum intensity projections. Insets are magnifications of individual nuclei. The scale bars represent $50 \mu \mathrm{m}$. 
TALE-deficient strain $X$. oryzae X11-5A (Triplett et al. 2011) for coimmunoprecipitation (co-IP) from inoculated Carolina Gold leaves (Fig. 4). Abilities of the tagged TALE and truncTALE to respectively trigger and suppress Xol-mediated resistance were confirmed (Supplementary Fig. S2). We also included a plasmid for expression of a second, untagged TALE (Tal3c from BLS256) and a plasmid for untagged Tal2h. By pairing the X11-5A transformants with each other or with the untransformed control strain, we were able to probe for Carolina Gold proteins interacting with the tagged TALE or truncTALE and for interactions of these proteins with each other or with the second TALE. Select combinations were inoculated to Nipponbare leaves for comparison. Inoculation was done by syringe infiltration, in 30 to 40 contiguous spots on each side of the leaf midrib. For each coinoculation, tissue was harvested at $48 \mathrm{~h}$ and was ground in liquid $\mathrm{N}_{2}$, then soluble extract was incubated with anti-FLAG agarose beads and was washed to immunopurify the tagged and interacting proteins. Immunoprecipitates were eluted, and an aliquot of each was subjected to Western blotting with anti-TALE antibody (Supplementary Fig. S3). The remainders were then resolved on a 4 to $20 \%$ sodium dodecyl sulfate-polyacrylamide electrophoresis gel, eluates from gel slices containing proteins between approximately 60 and $300 \mathrm{kDa}$ (Supplementary Fig. S4) were digested, and the peptides analyzed by mass spectrometry. Proteins were considered present in a sample if at least three peptides mapped uniquely to any of the pertinent annotated genomes searched: the $X$. oryzae X11-5A genome (Triplett et al. 2011) plus the TALE or TALEs or truncTALE being expressed, the Nipponbare genome (MSU 7 [Kawahara et al. 2013]), and the Carolina Gold genome (Read et al. 2020). For the Carolina Gold genome, we reannotated using the RNA-seq data from
CFBP7331(EV), CFBP7331(p2h), and mock-inoculated plants cited earlier. We carried out the experiment twice.

In the Western blot for each experiment (Supplementary Fig. S3), we detected the tagged TALE or truncTALE in each corresponding sample, with the exception of a Tal1cFLAG/Tal3c/Nipponbare sample in the first experiment. No Tal3c or untagged Tal2h was detected in any sample. The mass spectrometry confirmed these observations, suggesting that neither TALEs with truncTALEs nor TALEs with other TALEs interact appreciably (Fig. 4). Xo1 was consistently detected in the Carolina Gold/Tal2h-FLAG samples, irrespective of any codelivered Tal1c or Tal3c and not in the Tal1c-FLAG samples or any other sample (Fig. 4). No other protein consistently copurified with Tal2h-FLAG or Tal1c-FLAG in either Carolina Gold or Nipponbare samples (Supplementary Dataset S1).

In summary, we have shown that i) an NLR protein gene at the Xol locus, harboring an integrated zfBED domain, is Xol; i) the $\mathrm{Xol}$-mediated response is more similar to those mediated by two other NLR resistance genes than it is to the response associated with TALE-specific transcriptional activation of an executor resistance gene; i) a truncTALE abolishes or dampens activation of defense-associated genes by Xol; iv) the Xol protein, like TALEs and truncTALEs, localizes to the nucleus, and v) Xo1 specifically coimmunoprecipitates from rice leaves with a pathogen-delivered, epitope-tagged truncTALE. Thus, $X o 1$ is an allele or paralog of Xal, and suppression of Xo1 function by a truncTALE is likely the result of physical interaction between the resistance protein and the effector. The latter prediction is consistent with the Xo1 DEG profile during suppression by Tal2h, which suggested that Tal2h functions early in the defense cascade, perhaps by blocking TALE recognition by Xo1.

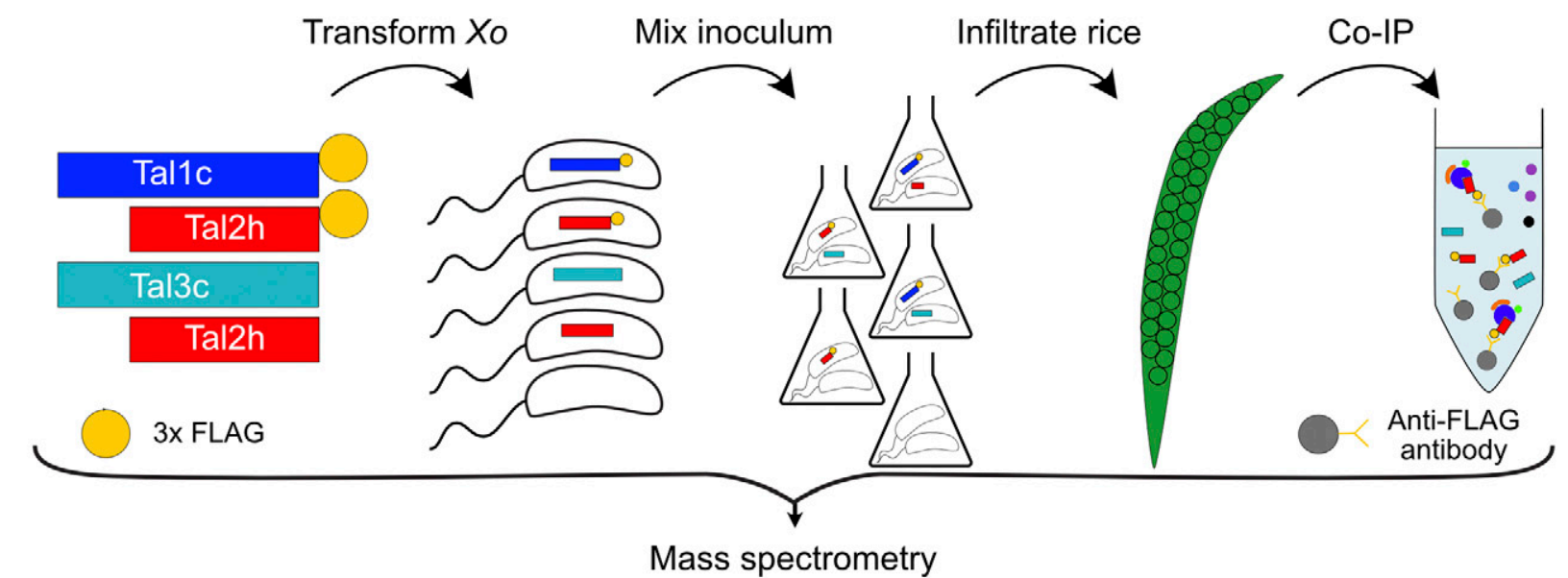

\begin{tabular}{|c|c|c|c|c|c|c|c|c|c|c|c|c|c|c|c|c|}
\hline & \multicolumn{10}{|c|}{ Carolina Gold } & \multicolumn{6}{|c|}{ Nipponbare } \\
\hline \multirow{2}{*}{ Input } & & & \multicolumn{2}{|c|}{\begin{tabular}{|l|} 
Tal2h \\
\end{tabular}} & \multirow{2}{*}{\multicolumn{2}{|c|}{\begin{tabular}{|l|} 
Tal2h \\
Tal3c
\end{tabular}}} & \multicolumn{2}{|c|}{$\overline{\text { Tal1c }}$} & \multicolumn{2}{|c|}{$\overline{\text { Tal1c }}$} & & & \multicolumn{2}{|c|}{\begin{tabular}{|l|} 
Tal2h \\
\end{tabular}} & \multicolumn{2}{|c|}{$\overline{\text { Tal1c }}$} \\
\hline & & & & & & & & & & & & & & & & \\
\hline Experiment & $\# 1$ & $\# 2$ & $\# 1$ & $\# 2$ & $\# 1$ & $\# 2$ & $\# 1$ & $\# 2$ & $\# 1$ & $\# 2$ & $\# 1$ & $\# 2$ & $\# 1$ & $\# 2$ & $\# 1$ & $\# 2$ \\
\hline Tal2h & - & - & 5 & 16 & 7 & 13 & - & - & - & - & - & - & 8 & 15 & - & - \\
\hline Tal3c & - & - & - & - & - & - & - & - & - & - & - & - & - & - & - & - \\
\hline Tal1c & - & - & - & - & - & - & 5 & 14 & 7 & 9 & - & - & - & - & - & 11 \\
\hline Xo1 & - & - & 4 & 6 & 3 & 6 & - & - & - & - & - & - & - & - & - & - \\
\hline
\end{tabular}

Fig. 4. Xo1 coimmunoprecipitates with Tal2h. Top, strategy used for coimmunoprecipitation (co-IP) of truncated transcription activator-like effectors (truncTALEs) Tal2h or Tallc and any interactors. Plasmid-borne expression constructs for Tal2h or Tallc with a C-terminal $3 \times$ FLAG tag as well as untagged Tal2h and a second TALE, Tal3c, were introduced into Xanthomonas oryzae X11-5A. Paired combinations of the transformants with each other or with the untransformed control strain or the control strain alone were coinfiltrated into leaves of rice varieties Carolina Gold and Nipponbare at a final optical density at $600 \mathrm{~nm}$ of 0.5 for each transformant. Samples were collected $48 \mathrm{~h}$ after inoculation, were ground, and were sonicated before co-IP using anti-FLAG agarose beads. After elution and sodium dodecyl sulfate-polyacrylamide gel electrophoresis separation, proteins between approximately 60 and 300 kDa were eluted, digested, and analyzed by mass spectrometry. The experiment was conducted twice. Bottom, co-IP results. For each immunoprecipitate, the numbers of unique peptides detected that matched Tal2h, Tal3c, Tal1c, or Xo1 in each experiment are shown. "-" indicates that $\leq 2$ unique peptides were detected. 
Whether the interaction between Tal2h and Xo1 is direct or indirect is not certain, but the fact that no other protein was detected consistently that coimmunoprecipitated with Tal2h and Xo1 suggests the interaction is direct. It is tempting to speculate also that TALEs trigger Xo1-mediated resistance by direct interaction with the protein and that truncTALEs function by disrupting the association. Though Tallc did not pull down Xo1, this might be explained by its lower apparent abundance, based on the Western blots. Tal1c might interact weakly or transiently with Xo1, or any complex of the proteins in the plant cells may have begun to degrade with the developing $\mathrm{HR}$ at the 48 -h timepoint sampled. It is also possible that Tal2h interacts with TALEs and masks them from the resistance protein, but both our co-IP results and the fact that Tal2h does not impact TALE activation of the OsSULTR3;6 susceptibility gene suggest that this is not the case. An alternative hypothesis is that Xo1 recognition of TALEs is not mediated by a direct interaction between the two proteins.

The results presented constitute an important step toward understanding how Xo1 works and how its function can be suppressed by the pathogen. An immediate next step toward determining the relationship of the interaction to defense suppression might be a structure function analysis of the interaction to determine the portion or portions of Xo1 and Tal2h involved. For Xo1, the leucine-rich repeat (LRR) may be the determinative interacting domain. Our previous comparison of the motifs present in $\mathrm{Xo1}_{11}, \mathrm{Xa1}$, and the closest Nipponbare homolog $\left(\mathrm{Nb}-\mathrm{xo}_{5}\right.$, which is expressed) revealed that the zfBED and coiled-coil domains are identical and the NB-ARC domains nearly so (Read et al. 2020). In contrast, the LRR domain of $\mathrm{Nb}$-xo $1_{5}$ differs markedly from those of Xo1 and $\mathrm{Xa1}$, which, with the exception of an additional repeat in Xa1, are very similar. Supporting this hypothesis, differences in the LRR determine the pathogen race specificities of some flax rust resistance genes (Ellis et al. 1999). More broadly, the ability of tagged Tal2h to pull down Xo1 suggests that effector co-IP may be an effective approach to characterizing pathogen recognition mechanisms of other resistance proteins or for identifying a resistance gene de novo.

While this paper was under review, Ji and colleagues ( $\mathrm{Ji}$ et al. 2020) reported the cloning and functional characterization of several $\mathrm{Xal}$ homologs, which also demonstrated that $X o 1_{11}$ is Xol.

\section{ACKNOWLEDGMENTS}

The authors thank M. Carter and B. Szurek for critical reading of the manuscript, M. Willmann and the Plant Transformation Facility of Cornell's School of Integrative Plant Science for carrying out the rice transformation, S. Harrington and S. McCouch for assistance growing the regenerants, and R. Bhawal and E. Anderson at the Proteomics Facility of the Biotechnology Resource Center at the Cornell University's Institute of Biotechnology (BRC) for conducting the mass spectrometry. Confocal microscopy was carried out at the BRC's Imaging Facility.

\section{LITERATURE CITED}

Bogdanove, A. J., Schornack, S., and Lahaye, T. 2010. TAL effectors: Finding plant genes for disease and defense. Curr. Opin. Plant Biol. 13: 394-401.

Caplan, J. L., Mamillapalli, P., Burch-Smith, T. M., Czymmek, K., and Dinesh-Kumar, S. P. 2008. Chloroplastic protein NRIP1 mediates innate immune receptor recognition of a viral effector. Cell 132:449-462.

Cernadas, R. A., Doyle, E. L., Niño-Liu, D. O., Wilkins, K. E., Bancroft, T., Wang, L., Schmidt, C. L., Caldo, R., Yang, B., White, F. F., Nettleton, D., Wise, R. P., and Bogdanove, A. J. 2014. Code-assisted discovery of TAL effector targets in bacterial leaf streak of rice reveals contrast with bacterial blight and a novel susceptibility gene. PLoS Pathog. 10: e1003972.
Cheng, Y. T., Germain, H., Wiermer, M., Bi, D., Xu, F., García, A. V., Wirthmueller, L., Després, C., Parker, J. E., Zhang, Y., and Li, X. 2009 Nuclear pore complex component MOS7/Nup88 is required for innate immunity and nuclear accumulation of defense regulators in Arabidopsis. Plant Cell 21:2503-2516.

Cui, H., Tsuda, K., and Parker, J. E. 2015. Effector-triggered immunity: From pathogen perception to robust defense. Annu. Rev. Plant Biol. 66: 487-511.

Ellis, J. G., Lawrence, G. J., Luck, J. E., and Dodds, P. N. 1999 Identification of regions in alleles of the flax rust resistance gene $L$ that determine differences in gene-for-gene specificity. Plant Cell 11: 495-506.

Hutin, M., Pérez-Quintero, A. L., Lopez, C., and Szurek, B. 2015. MorTAL Kombat: The story of defense against TAL effectors through loss-ofsusceptibility. Front. Plant Sci. 6:535.

Ji, C., Ji, Z., Liu, B., Cheng, H., Liu, H., Liu, S., Yang, B., and Chen, G. 2020. Xal allelic $R$ genes activate rice blight resistance suppressed by interfering TAL effectors. Plant Communications 1:100087.

Ji, Z., Ji, C., Liu, B., Zou, L., Chen, G., and Yang, B. 2016. Interfering TAL effectors of Xanthomonas oryzae neutralize $R$-gene-mediated plant disease resistance. Nat. Commun. 7:13435.

Kawahara, Y., de la Bastide, M., Hamilton, J. P., Kanamori, H., McCombie, W. R., Ouyang, S., Schwartz, D. C., Tanaka, T., Wu, J., Zhou, S., Childs, K. L., Davidson, R. M., Lin, H., Quesada-Ocampo, L., Vaillancourt, B., Sakai, H., Lee, S. S., Kim, J., Numa, H., Itoh, T., Buell, C. R., and Matsumoto, T. 2013. Improvement of the Oryza sativa Nipponbare reference genome using next generation sequence and optical map data. Rice (N. Y.) 6:4.

Lolle, S., Stevens, D., and Coaker, G. 2020. Plant NLR-triggered immunity: From receptor activation to downstream signaling. Curr. Opin. Immunol. 62:99-105.

McClung, A., and Fjellstrom, R. 2010. Using molecular genetics as a tool to identify and refine "Carolina Gold". Pages 37-41 in: The Golden Seed: Writings on the History and Culture of Carolina Gold Rice, D. S. Shields, ed. Douglas W. Bostick for the Carolina Gold Rice Foundation, Charleston, S.C., U.S.A.

Perez-Quintero, A. L., and Szurek, B. 2019. A decade decoded: Spies and hackers in the history of TAL effectors research. Annu. Rev. Phytopathol. 57:459-481.

Read, A. C., Moscou, M. J., Zimin, A. V., Pertea, G., Meyer, R. S., Purugganan, M. D., Leach, J. E., Triplett, L. R., Salzberg, S. L., and Bogdanove, A. J. 2020. Genome assembly and characterization of a complex zfBED-NLR gene-containing disease resistance locus in Carolina Gold Select rice with Nanopore sequencing. PLoS Genet. 16: e1008571.

Read, A. C., Rinaldi, F. C., Hutin, M., He, Y. Q., Triplett, L. R., and Bogdanove, A. J. 2016. Suppression of Xol-mediated disease resistance in rice by a truncated, non-DNA-binding TAL effector of Xanthomonas oryzae. Front. Plant Sci. 7:1516.

Shen, Q. H., Saijo, Y., Mauch, S., Biskup, C., Bieri, S., Keller, B., Seki, H., Ulker, B., Somssich, I. E., and Schulze-Lefert, P. 2007. Nuclear activity of MLA immune receptors links isolate-specific and basal diseaseresistance responses. Science 315:1098-1103.

Tanabe, S., Yokotani, N., Nagata, T., Fujisawa, Y., Jiang, C., Abe, K., Ichikawa, H., Mitsuda, N., Ohme-Takagi, M., Nishizawa, Y., and Minami, E. 2014. Spatial regulation of defense-related genes revealed by expression analysis using dissected tissues of rice leaves inoculated with Magnaporthe oryzae. J. Plant Physiol. Pathol. 2:1000135.

Tariq, R., Wang, C., Qin, T., Xu, F., Tang, Y., Gao, Y., Ji, Z., and Zhao, K. 2018. Comparative transcriptome profiling of rice near-isogenic line carrying Xa23 under infection of Xanthomonas oryzae pv. oryzae. Int. J. Mol. Sci. 19:717.

Triplett, L. R., Cohen, S. P., Heffelfinger, C., Schmidt, C. L., Huerta, A. I., Tekete, C., Verdier, V., Bogdanove, A. J., and Leach, J. E. 2016. A resistance locus in the American heirloom rice variety Carolina Gold Select is triggered by TAL effectors with diverse predicted targets and is effective against African strains of Xanthomonas oryzae pv. oryzicola Plant J. 87:472-483.

Triplett, L. R., Hamilton, J. P., Buell, C. R., Tisserat, N. A., Verdier, V., Zink, F., and Leach, J. E. 2011. Genomic analysis of Xanthomonas oryzae isolates from rice grown in the United States reveals substantial divergence from known $X$. oryzae pathovars. Appl. Environ. Microbiol. 77:3930-3937.

Wilkins, K. E., Booher, N. J., Wang, L., and Bogdanove, A. J. 2015. TAL effectors and activation of predicted host targets distinguish Asian from African strains of the rice pathogen Xanthomonas oryzae pv. oryzicola while strict conservation suggests universal importance of five TAL effectors. Front. Plant Sci. 6:536. 
Wirthmueller, L., Zhang, Y., Jones, J. D., and Parker, J. E. 2007. Nuclear accumulation of the Arabidopsis immune receptor RPS4 is necessary for triggering EDS1-dependent defense. Curr. Biol. 17:2023-2029.

Xie, X. W., Yu, J., Xu, J. L., Zhou, Y. L., and Li, Z. K. 2007. [Introduction of a non-host gene Rxol cloned from maize resistant to rice bacterial leaf streak into rice varieties]. Sheng Wu Gong Cheng Xue Bao 23:607-611.

Yoshimura, S., Yamanouchi, U., Katayose, Y., Toki, S., Wang, Z. X., Kono, I., Kurata, N., Yano, M., Iwata, N., and Sasaki, T. 1998. Expression of
$\mathrm{Xa1}$, a bacterial blight-resistance gene in rice, is induced by bacterial inoculation. Proc. Natl. Acad. Sci. U.S.A. 95:1663-1668.

Zhang, J., Yin, Z., and White, F. 2015. TAL effectors and the executor $R$ genes. Front. Plant Sci. 6:641.

Zhou, Y. L., Xu, M. R., Zhao, M. F., Xie, X. W., Zhu, L. H., Fu, B. Y., and Li, Z. K. 2010. Genome-wide gene responses in a transgenic rice line carrying the maize resistance gene Rxol to the rice bacterial streak pathogen, Xanthomonas oryzae pv. oryzicola. BMC Genomics 11:78. 